\title{
Techniques for Production and Detection of $2^{3} S$ Positronium
}

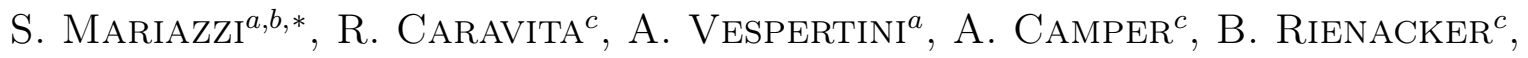

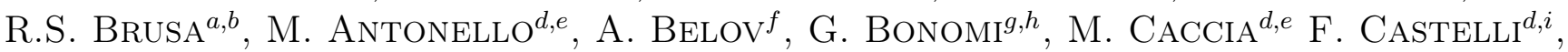
G. Cerchiari ${ }^{j}$, D. Comparat ${ }^{k}$, G. Consolati $^{l, d}$, A. Demetrio ${ }^{m, j}$, Di Noto ${ }^{n, o}$, M. Doser $^{c}$, M. Fanì ${ }^{c, n, o}$, J. Fesel ${ }^{c}$, S. Gerber ${ }^{c}$, A. Gligorova ${ }^{p}$, F. Guatieri ${ }^{a, b}$, P. Hackstock $^{p}$, S. Haider ${ }^{c}$, A. Hinterberger ${ }^{c}$, A. Kellerbauer $^{j}$, O. Khalidova ${ }^{c}$, D. Krasnicky $^{o}$, V. Lagomarsino ${ }^{n, o}$, P. Lebrun ${ }^{q}$, C. Malbrunot $^{c, p}$, V. MatveeV $^{f, r}$, S.R. Muller $^{m}$, G. Nebbia ${ }^{s}$, P. Nedelec ${ }^{q}$, M. Oberthaler ${ }^{m}$, E. Oswald ${ }^{c}$, D. Pagano ${ }^{g, h}$, L. Penasa $^{a, b}$ V. Petracek ${ }^{t}$, F. Prelz ${ }^{d}$, M. Prevedelli ${ }^{u}$, J. Robert ${ }^{k}$, O.M. Røhne ${ }^{v}$, A. Rotondi ${ }^{h, w}$,

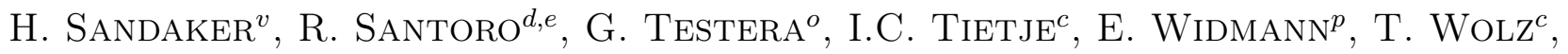
P. YZOMBARD ${ }^{j}$, C. ZIMmeR ${ }^{c, j, x}$ AND N. ZURLO ${ }^{h, y}$

${ }^{a}$ Department of Physics, University of Trento, via Sommarive 14, 38123 Povo, Trento, Italy ${ }^{b}$ TIFPA/INFN Trento, via Sommarive 14, 38123 Povo, Trento, Italy

${ }^{c}$ Physics Department, CERN, 1211 Geneva 23, Switzerland

${ }^{d}$ INFN Milano, via Celoria 16, 20133 Milano, Italy

${ }^{e}$ Department of Science, University of Insubria, Via Valleggio 11, 22100 Como, Italy

${ }^{f}$ Institute for Nuclear Research of the Russian Academy of Science, Moscow 117312, Russia

${ }^{g}$ Department of Mechanical and Industrial Engineering, University of Brescia, via Branze 38, 25123 Brescia, Italy

${ }^{h}$ INFN Pavia, via Bassi 6, 27100 Pavia, Italy

${ }^{i}$ Department of Physics "Aldo Pontremoli", University of Milano, via Celoria 16, 20133 Milano, Italy

${ }^{j}$ Max Planck Institute for Nuclear Physics, Saupfercheckweg 1, 69117 Heidelberg, Germany

${ }^{k}$ Laboratoire Aime Cotton, Université Paris-Sud, ENS Paris Saclay, CNRS, Université Paris-Saclay, 91405 Orsay Cedex, France

${ }^{l}$ Department of Aerospace Science and Technology, Politecnico di Milano, via La Masa 34, 20156 Milano, Italy

${ }^{m}$ Kirchhoff Institute for Physics, Heidelberg University, Im Neuenheimer Feld 227, 69120 Heidelberg, Germany

${ }^{n}$ Department of Physics, University of Genova, via Dodecaneso 33, 16146 Genova, Italy

${ }^{o}$ INFN Genova, via Dodecaneso 33, 16146 Genova, Italy

${ }^{p}$ Stefan Meyer Institute for Subatomic Physics, Austrian Academy of Sciences, Boltzmanngasse 3, 1090 Vienna, Austria

${ }^{q}$ Institute of Nuclear Physics, CNRS/IN2p3, University of Lyon 1, 69622 Villeurbanne, France

${ }^{r}$ Joint Institute for Nuclear Research, Dubna 141980, Russia

${ }^{s}$ INFN Padova, via Marzolo 8, 35131 Padova, Italy

${ }^{t}$ Czech Technical University, Prague, Brehova 7, 11519 Prague 1, Czech Republic

${ }^{u}$ University of Bologna, Viale Berti Pichat 6/2, 40126 Bologna, Italy

${ }^{v}$ Department of Physics, University of Oslo, Sem Sælandsvei 24, 0371 Oslo, Norway

${ }^{w}$ Department of Physics, University of Pavia, via Bassi 6, 27100 Pavia, Italy

${ }^{x}$ Department of Physics, Heidelberg University, Im Neuenheimer Feld 226, 69120 Heidelberg, Germany

${ }^{y}$ Department of Civil, Environmental, Architectural Engineering and Mathematics, University of Brescia, via Branze 43, 25123 Brescia, Italy

*corresponding author; e-mail: mariazzi@science.unitn.it 
In this work, we show recent measurements of $2^{3} S$ long-lived positronium production via spontaneous decay from the $3^{3} \mathrm{P}$ level. The possibility to tune the velocity of the $2^{3} S$ positronium, excited following this scheme, is presented. In the light of these results, we discuss the use of the $3^{3} P \rightarrow 2^{3} S$ transition to realize a monochromatic pulsed $2^{3} S$ positronium beam with low angular divergence. Preliminary tests of $2^{3} S$ beam production are presented. The possibility to overcome the natural $3^{3} \mathrm{P} \rightarrow 2^{3} S$ branching ratio via stimulated emission, and thus increasing the intensity of the $2^{3} S$ source, is also shown. A position-sensitive detector for a pulsed beam of positronium, with spatial resolution of $\approx 90 \mu \mathrm{m}$, is finally described in view of its possible application for the spatial characterization of the $2^{3} S$ beam.

DOI: 10.12693/APhysPolA.137.91

PACS/topics: 37.20.+j, 36.10.Dr, 78.70.Bj

\section{Introduction}

Positronium (Ps), the bound state of an electron and a positron $\left(\mathrm{e}^{+}\right)$, has a lifetime of $142 \mathrm{~ns}$ when it is in the triplet ground state $\left(1^{3} S\right)$. The lifetime of Ps increases up to $1140 \mathrm{~ns}$ when it is excited to the $2^{3} S$ level [1]. The relatively long lifetime and the scarce sensitivity to stray electric fields of this level [2] make $2^{3} S$ Ps a good candidate for deflectometry/interferometry measurements on a pure leptonic antimatter system with potential gravity sensitivity [3]. The production of a monochromatic beam of $2^{3} S$ Ps, with tunable velocity, could also pave the road to systematic studies of Ps quantum sticking and reflection on solid surfaces [4]. Finally, Ps in the $2^{3} S$ state has been recently proposed for the formation of a stable positronium Bose-Einstein condensate [5].

Several methods for producing $2^{3} S$ Ps have been demonstrated till now. Formation of fast $2^{3} S$ Ps with energies of several $\mathrm{eV}$ has been demonstrated via $\mathrm{e}^{+}$collisions with solid $[6-8]$ and gaseous targets $[9,10] .2^{3} S$ Ps atoms were also produced via Doppler-free two photon excitation of ground-state atoms of Ps desorbed from metallic surfaces [11-13] and from porous silicon [14]. $2^{3} S$ Ps production has been also demonstrated via single photon excitation of ground-state atoms to mixed $2^{3} S-2^{3} P$ in electric fields [2].

In this work, an alternative way to produce $2^{3} S$ Ps via the $3^{3} P \rightarrow 2^{3} S$ spontaneous decay $[15,16]$ is presented. We show that, with this method, it is possible to realize a monochromatic source of $2^{3} S$ with tunable velocity and to form a monochromatic $2^{3} S$ Ps beam [16]. Finally, a position-sensitive detector for a pulsed beam of Ps, with spatial resolution of around $90 \mu \mathrm{m}$ [17], is described.

\section{Experimental}

In the present experiments, an intense bunch of $\mathrm{e}^{+}$is prepared with the AEgIS positron system (see Ref. [18] for details) and implanted in a nanochanneled $\mathrm{Si}^{+} / \mathrm{Ps}$ converter $[19,20]$. A fraction of the formed Ps is emitted into the vacuum as a dense cloud of Ps in the $1^{3} S$ level. Ps is then excited to $n=3$ (precisely on the $3^{3} P$ state) with an UV laser pulse set at the resonance wavelength $\lambda_{\mathrm{UV}}=(205.045 \pm 0.005) \mathrm{nm}[21]$. The energy of the UV laser was $>60 \mu \mathrm{J}$, the bandwidth around $120 \mathrm{GHz}$, the full width half maximum (FWHM) of its temporal profile $\approx 1.5 \mathrm{~ns}$ and the FWHM of its spot $\approx 3.0-3.5 \mathrm{~mm}$. The

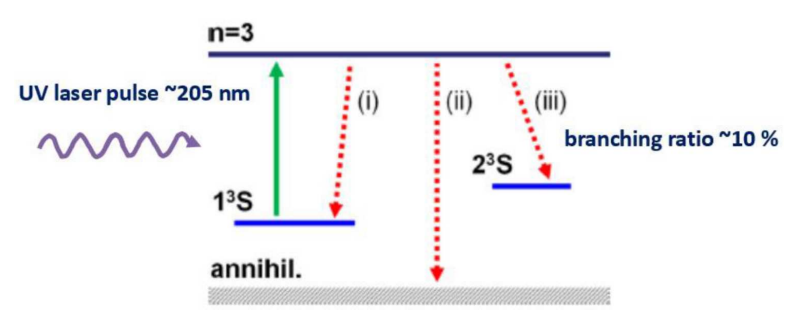

Fig. 1. Partial energy level diagram of Ps with the $n=3$ laser excitation and subsequent de-excitation processes to $1^{3} S$ level (i), annihilation (ii) and $2^{3} S$ level (iii).

laser setup is described in detail elsewhere [21, 22]. From the $n=3$ level, Ps spontaneously decays to $2^{3} S$ level (see Fig. 1) with a branching ratio of $\approx 10 \%[15,16]$. The present experiments were performed by placing the $\mathrm{e}^{+} /$Ps converter in a $0.0250 \mathrm{~T}$ magnetic field.

A $20 \times 25 \times 25 \mathrm{~mm}^{3} \mathrm{PbWO}_{4}$ scintillator, coupled to a Hamamatsu R11265-100 photomultiplier tube (PMT) and digitized by a HD4096 Teledyne LeCroy oscilloscope, was placed $40 \mathrm{~mm}$ above the $\mathrm{e}^{+} /$Ps converter and used to record the time distribution of gamma rays emitted by $\mathrm{e}^{+}$and Ps annihilations (single-shot positron annihilation lifetime spectroscopy spectrum, SSPALS). SSPALS spectra were acquired and analyzed, with the procedure described in Refs. $[15,16]$, with the goal to study the increase of the Ps lifetime due to the presence of atoms on the $2^{3} S$ level.

\section{Results and discussion}

\subsection{Production of monochromatic $2^{3} S$ Ps}

In Fig. 2 (main figure), we report SSPALS spectra of positronium into vacuum acquired with the UV laser on and off.

Both spectra show a prompt peak, given by the fast $2 \gamma$ annihilations of $\mathrm{e}^{+}$implanted in the converter, and a tail that is dominated by the $3 \gamma$ decay of $1^{3} S$ Ps emitted into vacuum. In the presence of $2^{3} S$ atoms, due to their long lifetime, a large fraction of atoms survives in-flight annihilation and reaches the experimental chamber walls, where it annihilates in $2 \gamma$. This produces a signal excess identifiable from the comparison of the SSPALS spectra with laser on and off. The amplitude of this excess can be estimated as $S=\left(f_{\text {off }}-f_{\text {on }}\right) / f_{\text {off }}$ (where $f_{\text {off }}$ and $f_{\text {on }}$ 


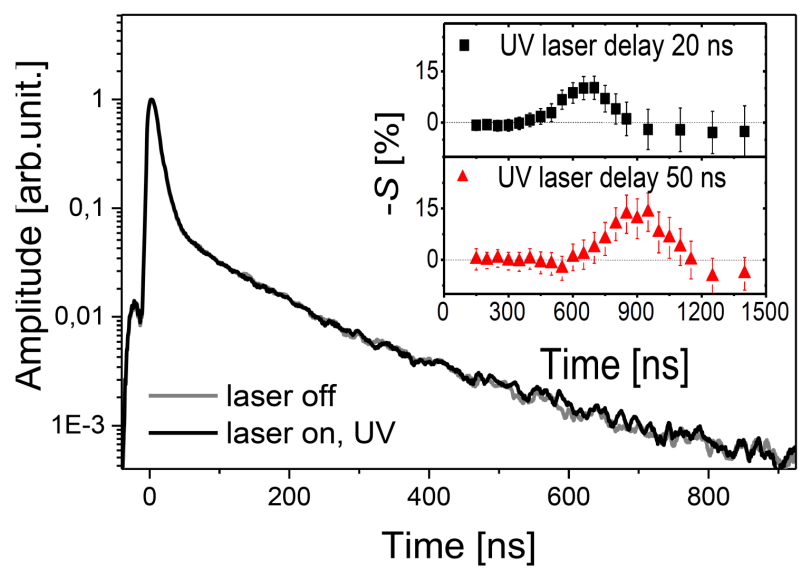

Fig. 2. (main figure) SSPALS spectra of Ps into vacuum with laser off (gray) and UV laser on (black) normalized to the prompt peak height. (inset) $-S$ parameter (see text) as a function of the time elapsed from the prompt peak in the case of UV laser delayed of 20 and $50 \mathrm{~ns}$.

are the areas below the spectra without and with the UV laser, respectively) and in the case of Fig. 2, it amounts to around $10 \%$, between 500 and $800 \mathrm{~ns}$ from the prompt peak [16].

The employed nanochanneled $\mathrm{Si}^{+} / \mathrm{Ps}$ converter emits $P_{s}$ in a broad distribution of velocities [20, 23]. More in detail, Ps emitted with low velocity spend more time in the nanochannels losing energy by interaction with the walls while fast Ps are emitted in a very short time (few ns) [24, 25]. The brief duration and the limited spatial dimension of the exciting UV laser pulse allow to address only the fraction of the emitted Ps in front of the target at the time when the laser is shot. Ps atoms with different velocities can be thus excited to the $3^{3} P$ level (subsequently decaying to the $2^{3} S$ level) varying the time at which the laser is shot [16].

In the inset of Fig. 2, we show two examples of $-S$ parameter evaluated as a function of the time elapsed from the prompt peak in time windows of $300 \mathrm{~ns}$ with steps of 50 ns starting from 150 ns for two different UV laser delay. The plot shows that the time position of the signal excess given by $2^{3} S$ annihilation on the chamber walls is progressively delayed by retarding the UV pulse. When the UV laser pulse is shot 20 ns after $\mathrm{e}^{+}$implantation, the $2^{3} S$ signal excess peaks at around $650-700 \mathrm{~ns}$ (Fig. 2 in the inset). This time increases to 900 ns when the UV pulse is delayed to 50 ns. We have observed that, at $65 \mathrm{~ns}$, the excess of annihilations disappears [16] pointing out that also very slow Ps emitted by the converter has already left the laser spot. By knowing the distance between the target and the chamber walls $(6 \mathrm{~cm}$ on average), the average velocity of the produced $2^{3} S$ Ps has been estimated to be $(1.0 \pm 0.1) \times 10^{5} \mathrm{~m} / \mathrm{s}$ and $(0.7 \pm 0.1) \times 10^{5} \mathrm{~m} / \mathrm{s}$, for an UV delay of 20 and $50 \mathrm{~ns}$, respectively [16].

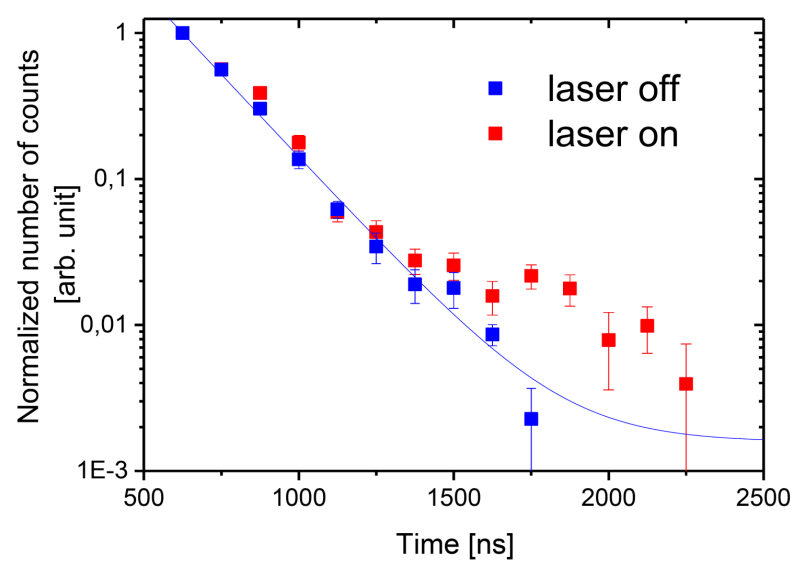

Fig. 3. Normalized number of gamma rays counted by the $\mathrm{LaBr}_{3}(\mathrm{Ce})$ scintillator in the stopper region $(30 \mathrm{~cm}$ far from the converter) with and without the laser pulse.

The continuous line is a guide for the eyes.

Given this monochromatic $2^{3} S$ Ps source, a beam of $2^{3} S$ can be produced by placing an iris of $1.6 \mathrm{~cm}$ of diameter at a distance of $10.8 \mathrm{~cm}$ from the target [26]. This iris selects a beam expanding within a solid angle of $\approx 17 \mathrm{msr}$. The beam within this angle has been let to freely expand up to a distance of $30 \mathrm{~cm}$ from the converter. At that distance a stopper for the beam has been placed and the gamma rays generated by the $2 \gamma$ annihilations of $2^{3} S$ Ps atoms on the stopper have been monitored by a $76.2 \times 76.2 \mathrm{~mm}^{2} \mathrm{LaBr}_{3}(\mathrm{Ce})$ scintillator, coupled to a PMT Hamamtsu R10.233-100-01. Thanks to its excellent energy resolution $(\approx 3 \%$ at $662 \mathrm{keV})$ and very high light yield $\left(\approx 160 \%\right.$ of $\mathrm{NaI}$ ) [27], $\mathrm{LaBr}_{3}(\mathrm{Ce})$ scintillator allows to distinguish individual $511 \mathrm{keV}$ generated by Ps pick-off annihilations and record them with high efficiency. In our experimental condition, this scintillator operated in single counting mode. Lead shields have been used to reduce the background signal given by gamma rays coming from the $\mathrm{e}^{+}$annihilation in the converter. The PMT was also shielded by $\mu$-metal foils from the magnetic fields generated from the positron transfer line [17].

In Fig. 3, we show a preliminary measurement of the time distribution of the $511 \mathrm{keV}$ gamma rays detected by the $\mathrm{LaBr}_{3}(\mathrm{Ce})$ scintillator in the stopper region in the case of laser off (no $2^{3} S$ production) and on $\left(2^{3} S\right.$ annihilations on the stopper). In the presence of the UV laser pulse (delay of $15 \mathrm{~ns}$ from the prompt peak), it is evident an increase in the number of the counts between 1600 and $2250 \mathrm{~ns}$ (corresponding to an average velocity of $\left.(1.6 \pm 0.4) \times 10^{5} \mathrm{~m} / \mathrm{s}\right)$.

The intensity of this collimated monochromatic beam (currently of the order of $10^{-6} 2^{3} S$ atoms per implanted $\mathrm{e}^{+}$at a distance of $30 \mathrm{~cm}$ from the source [26]) is strongly limited by the low branching ratio of the $3^{3} \mathrm{P} \rightarrow 2^{3} \mathrm{~S}$ spontaneous decay. Tests of stimulated $3^{3} P \rightarrow 2^{3} S$ transition have been performed by adding an IR pulse at $\approx 1312 \mathrm{~nm}$ simultaneous with the UV pulse at $205 \mathrm{~nm}$. 
In presence of the stimulating IR laser pulse, an increase in the number of produced $2^{3} S$ atoms of a factor $\approx 3$ has been observed (the interested reader is referred to [28]).

\subsection{Position-sensitive detector for a pulsed beam of slow Ps}

In view of the possible application in the spatial characterization of $2^{3} S$ Ps beams, a position-sensitive detector for a pulsed beam of positronium has been developed [17, 29]. The detector is based on a microchannel plate (MCP)-phosphor screen assembly, housed in a $1 \mathrm{~T}$ magnetic field, and an ionization laser with ns-long pulse. Photo- $\mathrm{e}^{+}$and photo- $\mathrm{e}^{-}$, freed by the Ps ionization, are bound to their magnetic field line, thus conserving the original positronium position in the plane transverse to the magnetic field ( $x-y$ plane) at the ionization instant. By negatively biasing the MCP front face, electrons are repelled while positrons are attracted and their spatial distribution is imaged.

The spatial resolution of the detector system has been determined by photoionizing a Ps cloud whose view of the MCP was partially shadowed by a sharp edge (Fig. 4).

The dark region in the upper part of the picture (negative $x$ ) is due to the shadow of the edge while the bright part below (positive $x$ ) is given by photo- $\mathrm{e}^{+}$able to reach the MCP assembly. The sharp edge generates a step transition between the two regions that is smoothed by the spatial resolution of the detector. The intensity of the image has been integrated in $y$ in the marked region. The obtained plot of the intensity $I$ as a function of the $x$-position is reported in the left part of Fig. 4. The transition from the no-signal to the maximum intensity region has been found to occur in $(88 \pm 5) \mu \mathrm{m}$ (FWHM of the derivative of $I, \mathrm{~d} I / \mathrm{d} x$ ) indicating that the resolution of this position sensitive detector for Ps is of the order of $90 \mu \mathrm{m}$ [17].

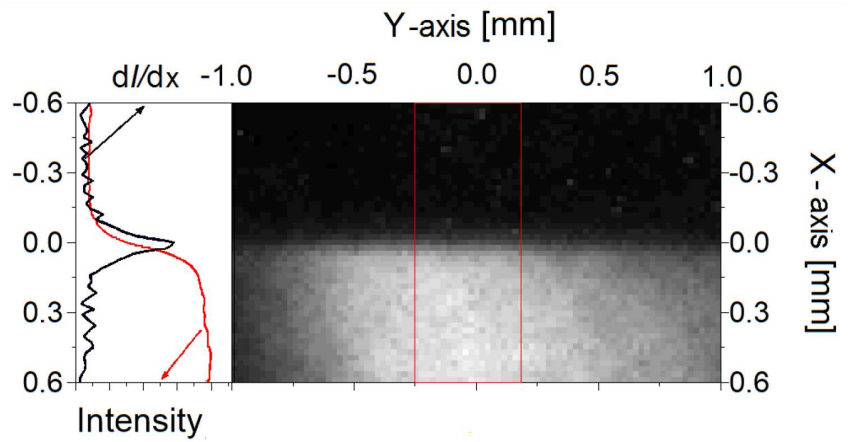

Fig. 4. Spatial distribution in the plane transverse to the $1.0 \mathrm{~T}$ field of photo-e ${ }^{+}$produced by the Ps photoionization. The bright part (positive- $x$ ) is given by photo-e ${ }^{+}$able to reach the MCP assembly while the dark (negative- $x$ ) is due to the presence of a sharp edge used to partially shadow the MCP (see text for details). The intensity $I$ profile along the $x$-axis, calculated in the indicated perimeter, is shown on the left of the image. The derivative of the intensity $\mathrm{d} I / \mathrm{d} x$ is also reported.

\section{Conclusions}

In this work, we have demonstrated the possibility to realize a pulsed monochromatic source of $2^{3} S$ positronium via spontaneous decay from $3^{3} P$ level previously populated by exciting $1^{3} S$ Ps with an UV laser pulse at around $205 \mathrm{~nm}$. The possibility to tune the velocity of the produced $2^{3} S$ Ps in the range of high- $10^{4}$ low $-10^{5} \mathrm{~m} / \mathrm{s}$ has been shown. A low divergence $2^{3} S$ beam $(17 \mathrm{msr}$ ) has then been selected by employing an iris. The $2^{3} S$ beam within this solid angle has been let to freely expand up to a distance of $30 \mathrm{~cm}$ from the converter. The annihilations of the $2^{3} S$ atoms on a stopper have been detected with a $\mathrm{LaBr}_{3}(\mathrm{Ce})$ scintillator. The possibility to increase the intensity of the $2^{3} S$ source by employing a scheme of $3^{3} P \rightarrow 2^{3} S$ stimulated emission has been also shown.

Finally, we have described a position-sensitive detector for a pulsed beam of Ps based on the imaging of photo$\mathrm{e}^{+}$freed by photoionization of positronium in a strong magnetic field. A spatial resolution of around $90 \mu \mathrm{m}$ has been demonstrated. We plan to use this detection scheme for the spatial characterization of the $2^{3} S$ beam.

\section{References}

[1] D.B. Cassidy, Eur. Phys. J. D 72, 53 (2018).

[2] A.M. Alonso, S.D. Hogan, D.B. Cassidy, Phys. Rev. A 95, 033408 (2017).

[3] M. Oberthaler, Nucl. Instrum. Methods Phys. Res. B 192, 129 (2002).

[4] R. Saniz, B. Barbiellini, P.M. Platzman, A.J. Freeman, Phys. Rev. Lett. 99, 096101 (2007).

[5] Y. Zhang, M.S. Wu, J.Y. Zhang, Y. Qian, X. Gao, K. Varga, arXiv:1903.08353 (2019).

[6] K.F. Canter, A.P.J. Mills, S. Berko, Phys. Rev. Lett. 34, 177 (1975).

[7] T.D. Steiger, R.S. Conti, Phys. Rev. A 45, 2744 (1992).

[8] D.J. Day, M. Charlton, G. Laricchia, J. Phys. B 34 3617 (2001).

[9] G. Laricchia, M. Charlton, G. Clark, T.C. Griffith, Phys. Lett. A 109, 97 (1985).

[10] D.J. Murtagh, D.A. Cooke, G. Laricchia, Phys. Rev. Lett. 102, 133202 (2009).

[11] S. Chu, A.P. Mills, Phys. Rev. Lett. 48, 1333 (1982).

[12] M.S. Fee, A.P. Mills, S. Chu, E.D. Shaw, K. Danzmann, R.J. Chichester, D.M. Zuckerman, Phys. Rev. Lett. 70, 1397 (1993).

[13] M.S. Fee, S. Chu, A.P.J. Mills, R.J. Chichester, D.M. Zuckerman, E.D. Shaw, K. Danzmann, Phys. Rev. A 48, 192 (1993).

[14] D.A. Cooke, P. Crivelli, J. Alnis, A. Antognini, B. Brown, S. Friedreich, A. Gabard, T.W. Haensch, K. Kirch, A. Rubbia, V. Vrankovic, Hyperfine Interact. 233, 67 (2015).

[15] S. Aghion et al. (AEgIS collaboration), Phys. Rev. A 98, 013402 (2018). 
[16] C. Amsler et al. (AEgIS collaboration), Phys. Rev. A 99, 033405 (2019).

[17] S. Aghion, et al. (AEgIS collaboration), Nucl. Instrum. Meth. Phys. Res. B 457, 44 (2019).

[18] S. Aghion, et al. (AEgIS collaboration), Nucl. Instrum. Meth. Phys. Res. B 362, 86 (2015).

[19] S. Mariazzi, P. Bettotti, S. Larcheri, L. Toniutti, R.S. Brusa, Phys. Rev. B 81, 235418 (2010).

[20] S. Mariazzi, P. Bettotti, R.S. Brusa, Phys. Rev. Lett. 104, 243401 (2010).

[21] S. Aghion, et al. (AEgIS collaboration), Phys. Rev. A 94, 012507 (2016).

[22] S. Cialdi, I. Boscolo, F. Castelli, F. Villa, G. Ferrari, M. Giammarchi, Nucl. Instrum. Methods Phys. Res. B 269, 1527 (2011).
[23] F. Guatieri, Ph.D. Thesis, University of Trento, 2018.

[24] D.B. Cassidy, T.H. Hisakado, V.E. Meligne, H.W.K. Tom, A.P. Mills, Phys. Rev. A 82, 052511 (2010).

[25] S. Mariazzi, L. Di Noto, G. Nebbia, R.S. Brusa, J. Phys. Conf. Ser. 618, 012039 (2015).

[26] M. Antonello, et al. (AEgIS collaboration), in preparation.

[27] B.D. Milbrath, B.J. Choate, J.E. Fast, W.K. Hensley, R.T. Kouzes, J.E. Schweppe, Nucl. Instrum. Methods Phys. Res. A 572, 774 (2007).

[28] M. Antonello et al., (AEgIS collabration) Phys. Rev. A 100, 063414 (2019).

[29] A. Camper, et al. (AEgIS collaboration), EPJ Web Conf. 198, 00004 (2019). 